\title{
O uso de fitoterápicos na gestação: Gengibre (Zingiberofficinale) e seus benefícios
}

\author{
The use of herbal medicines during pregnancy: ginger (Zingiberofficinale) and its benefits \\ El uso de medicamentos a base de hierbas durante el embarazo: jengibre (Zingiberofficinale) y sus \\ beneficios
}

Recebido: 29/10/2021 | Revisado: 07/11/2021 | Aceito: 09/11/2021 | Publicado: 14/11/2021

\author{
Ana Luiza de Lima Cavalcanti \\ ORCID: https://orcid.org/0000-0003-3242-4177 \\ Centro Universitário Unifavip, Brasil \\ E-mail:ana luiza-sc@hotmail.com \\ Débora Mari Brito do Nascimento \\ ORCID: https://orcid.org/0000-0003-3071-9588 \\ Centro Universitário Unifavip, Brasil \\ E-mail:deboramari77@gmail.com \\ Tibério Cesar Lima de Vasconcelos \\ ORCID: https://orcid.org/0000-0001-7177-0561 \\ Centro Universitário Unifavip, Brasil \\ E-mail:tiberio.vasconcelos@professores.unifavip.edu.br
}

\begin{abstract}
Resumo
O uso de extratos naturais das plantas é uma prática bastante antiga e seu uso na medicina popular foi propagado de cultura para cultura. Durante o período gestacional é quando muitas mulheres acabam procurando o uso de algumas plantas para aliviar os sintomas que surgem nesse período, por isso buscou-se analisar os benefícios da utilização do gengibre (Zingiberofficinale) frente aos sintomas do período gravídico. O gengibre que é uma planta bastante conhecida e vem sendo muito utilizada, sendo assim, serviu de estudo pelo seu poderoso potencial antiemético. Diante disso, o objetivo desse estudo foi realizar uma revisão integrativa da literatura, ressaltando a eficácia do gengibre no alívio de sintomas causados pela gestação. Como respaldo para a pesquisa foram utilizados SciELO, ScienceDirect, EBSCO e PubMed, e os materiais analisados foram os publicados entre os anos de 2011 à2021.Conclui-se que com os dados científicos obtidos, o uso do gengibre apresentou resultados satisfatórios que apontam uma redução significativa dos sintomas de náuseas e vômitos durante a gestação.
\end{abstract}

Palavras-chave: Fitoterápicos; Zingiberofficinale; Gengibre; Gravidez.

\begin{abstract}
The use of natural plant extracts is a very old practice and its use in folk medicine has spread from culture to culture. During the gestational period is when many women end up looking for the use of some plants to alleviate the symptoms that arise during this period, so we sought to analyze the benefits of using ginger (Zingiberofficinale) against the symptoms of the pregnancy period. Ginger, which is a well-known plant and has been widely used, therefore, it served as a study for its powerful antiemetic potential. Therefore, the aim of this study was to carry out an integrative literature review, emphasizing the effectiveness of ginger in relieving symptoms caused by pregnancy. To support the research, SciELO, ScienceDirect, EBSCO and PubMed were used, and the analyzed materials were published between the years 2011 to 2021. It is concluded that with the scientific data obtained, the use of ginger presented satisfactory results that point to a significant reduction in the symptoms of náusea and vomiting during pregnancy.
\end{abstract}

Keywords: Phytotherapy; Zingiberofficinale; Ginger; Pregnancy.

\section{Resumen}

El uso de extratos de plantas naturales es una práctica muy antigua y su uso en la medicina popular se ha extendido de una cultura a otra. Durante el período gestacional es cuando muchas mujeres terminan buscando el uso de algunas plantas para paliar los síntomas que surgen durante este período, por lo que buscamos analizar los beneficios de usar jengibre (Zingiberofficinale) frente a los síntomas del período de embarazo. El jengibre, que es una planta muy conocida y muy utilizada, sirvió de estudio por su potente potencial antiemético. Por ello, el objetivo de este estudio fue realizar una revisión integradora de la literatura, destacando la efectividad del jengibre en el alivio de los síntomas provocados por el embarazo, como apoyo a la investigación se utilizaron SciELO, ScienceDirect, EBSCO y PubMed, y los materiales analizados fueron publicados entre los años 2011 a 2021. Se concluye que con los datos científicos obtenidos, el uso de jengibre presentó resultados satisfactorios que apuntan a una reducción significativa de los síntomas de náuseas y vómitos durante el embarazo.

Palabras clave: Hierbas medicinales; Zingiberofficinale; Jengibre; Embarazo. 


\section{Introdução}

O uso de fitoterápicos pela sociedade é uma prática bastante antiga, que nunca deixou de ser utilizada, mesmo com todas as tecnologias que surgiram ao longo dos anos. Desde muito cedo, o medicamento fitoterápico é utilizado para tratar diversos problemas de saúde e até hoje, em muitos lugares do mundo, se fazem uso desses fitoterápicos como parte da sua medicina (Gomes, 2018).

Com a justificativa de que é mais segura e menos tóxica, a fitoterapia vem ganhando popularidade no mundo, porém, no caso de algumas plantas não se pode garantir sua eficiência, pelo fato de que ainda não houveram estudos que comprovem sua eficácia e segurança. Nesses casos, a fitoterapia pode oferecer problemas a saúde do indivíduo, principalmente se forem crianças, gestantes e idosos (Cardoso \& Amaral, 2017).

Durante a gravidez, muitas mulheres acabam procurando o uso de algumas plantas e fitoterápicos para o alívio de sintomas que surgem nesta fase da vida. Esta procura está integralmente associada a crença de que não faz mal ao feto e nem a mãe por ser um produto natural, e que apresentam menos riscos que outros medicamentos. Contudo, nem sempre é assim (Trabace, et al., 2015).

Quando este fato recai para a utilização de fitoterápicos pelas gestantes, torna-se ainda mais preocupante pois, buscar por uma planta determinada medicinal sem indicação de um profissional ou pelo desconhecimento cultural, pode ocasionar efeitos indesejados (Cardoso \& Amaral, 2017).

Sintomas comuns durante a gravidez, náuseas e vômitos ocorrem em até 80-85\% das mulheres e sua gravidade varia entre as grávidas. De acordo com algumas normas de orientações médicas, as preparações a base do gengibre têm sido bastantes utilizadas como terapias alternativas aos fármacos convencionais. O gengibre é também utilizado como um antiemético em outras situações clínicas como no pós-operatório, na quimioterapia e em enjôos de movimento (Amorin, et al., 2013).

Zingiberofficinale, Roscoe ou gengibre como é popularmente conhecida, é uma planta cujo rizoma tem finalidade tanto alimentar, como industrial e medicinal (Afzal, et al., 2001; Chrubasik, et al., 2005). Em relação a suas atividades farmacológicas o gengibre possui grande propriedades anti-inflamatórias (Flynn, et al., 1968, Grzanna, et al., 2005; Thomson, et al., 2002; Ojewole, 2006) antimicrobianas, (Chrubasik, et al., 2005; Ficker, et al., 2003, Iqbal, et al., 2006) e também é usada como tratamento para distúrbios gastrointestinais.

Suas propriedades comprovam que a atualização do gengibre durante o período gestacional é eficaz no tratamento de náuseas e vômitos (Duarte, et al., 2017). Fazer a utilização do gengibre por via oral pode reduzir a gravidade das náuseas e dos vômitos nos enjôos matinais (Ding, et al., 2013). Ademais, o presente estudo tem como objetivo ressaltar a eficácia do gengibre (Zingiberofficinale) no alívio de sintomas causadas pela gravidez.

\section{Metodologia}

O estudo trata-se de uma revisão bibliográfica integrativa da literatura, com abordagem qualitativa sobre o uso de fitoterápicos na gestação, falando mais especificamente do gengibre (Zingiberofficinale) e seus benefícios. Como respaldo para a pesquisa, foram utilizados SciELO, ScienceDirect, EBSCO e PubMed. Logo, foram obedecidas as seguintes etapas na revisão: identificação do tema; estabelecimento de critérios para inclusão e exclusão de estudos; identificação dos estudos nas bases científicas; avaliação dos dados e análise e interpretação dos resultados da revisão, evidenciando o conhecimento obtido (Pereira, et al., 2018).

Como critérios de inclusão, buscaram-se artigos que abordassem o tema da pesquisa onde foram inclusos livros, periódicos e documentos sem distinção de área, que tivessem como descritores gengibre, (Zingiberofficinale), ginger, gravidez 
e fitoterápicos. Para critério de exclusão, foram descartados os artigos que não se enquadravam no tema ou que não atenderam a temática principal. Os materiais analisados foram os publicados entre os anos de 2011-2021.

\section{Resultados e Discussão}

Durante o primeiro trimestre de gestação, é comum que algumas mulheres se queixem de sentir náuseas e enjôos devido a adaptação do corpo à novas mudanças que irão acontecer, sendo relatados em geral, por 60-80\% das gestantes. Normalmente, estes sinais aparecem entre a $4^{\circ}$ e $8^{\circ}$ semana de gestação e desaparecem por volta da $16^{\circ}$ semana, podendo variar de mulher para mulher (Holand, 2017; Duarte, et al., 2017).

Esses sintomas estão relacionados aos altos índices do hormônio gonadotrófico humano, tem seu ápice no sangue materno por volta da $12^{\circ}$ semana de gestação, o que está relacionado com a melhora dos sintomas depois desse período. É nessa etapa da gestação que muitas mulheres procuram o uso de plantas medicinais para aliviar os sintomas (Holand, 2017; Duarte, et al., 2017).

O uso de fitoterápicos como o gengibre, por exemplo, é normalmente utilizado para tratar doenças gastrointestinais como diarréia, náuseas, desconfortos abdominais além de ser uma especiaria bastante utilizada nas cozinhas brasileiras, realçando o sabor dos alimentos (Prato, 2010).

O Zingiberofficinale conhecido popularmente como gengibre, é uma planta cujo rizoma tem um grande emprego alimentar, industrial e medicinal. Com relação as propriedades farmacológicas, o gengibre apresenta grande espectro de ação, possuindo atividades anti-inflamatórias e antimicrobianas, além de poder ser utilizado para tratamento de distúrbios gastrointestinais inclusive, distúrbios presentes durante o período de gravidez. Diante de alguns estudos, o gengibre se mostrou eficaz no tratamento de náuseas e vômitos, durante a gestação (Duarte, et al., 2017).

Foi possível visualizar nas pesquisas e nos estudos realizados, que as substancias gingerol e shogal (compostos não voláteis), presentes no gengibre, são as principais substâncias responsáveis pelas características organolépticas e terapêuticas do gengibre, além de possuir propriedades atividade anti-inflamatória e antiemética (que inibem o vômito) (Hemkemeier, et al., 2018).

Na Tabela 1 são descritas algumas das atividades biológicas destes respectivos compostos ativos:

Tabela 1. Atividades biológicas dos compostos ativos do gengibre. (Conceição, 2019).

\begin{tabular}{|c|c|}
\hline COMPOSTO ATIVO & ATIVIDADES BIOLOGICAS \\
\hline Gingerol & $\begin{array}{c}\text { Atividade antioxidante; } \\
\end{array}$ \\
& $\begin{array}{c}\text { Atividade antitumoral através da indução de apoptose; } \\
\text { Atividade anti-inflamatória e analgésica; } \\
\text { Atividade antimicrobiana; } \\
\text { Atividade protetora hepática. }\end{array}$ \\
\hline Shogal & $\begin{array}{c}\text { Atividade antioxidante e anti-inflamatória; } \\
\text { Atividade anti-proliferação e antiinvasão. }\end{array}$ \\
\hline Zingerona & $\begin{array}{c}\text { Atividade antioxidante; } \\
\text { Ação anti-inflamatória; } \\
\text { Atividade antibacteriana. }\end{array}$ \\
\hline Paradol & Atividade antioxidante e anticancerígena; Atividade \\
& antimicrobiana \\
\hline Flavonóides de gengibre & Atividade antioxidante \\
\hline
\end{tabular}

Fonte: Adaptado (Conceição, 2019). 
De acordo com os artigos estudados e que abordaram o uso do gengibre na gravidez, foi possível observar que o gengibre apresenta uma ótima efetividade quanto a aliviar os sintomas de náuseas e vômitos na gravidez, ao qual tem se demonstrado uma alternativa segura e de baixo custo (Lete, et al., 2016).

De acordo com os estudos de Viljoen, et al., (2014) e Thomson, et al., (2014) o uso do gengibre durante a gestação, foi usado em diferentes dosagens e diferentes grupos de controle (placebo, vitamina B6, metoclopramida), todos os experimentos demonstraram ação semelhante a metoclopramida e a vitamina B6 na redução das náuseas, porém não foi possível observar a relação da diminuição de vômitos.

Já em outro estudo foi possível observar que o gengibre se mostrou mais eficaz do que o placebo, comparável coma a vitamina B6 e também comparável ao dimenidrinato, visto que o gengibre pode funcionar mais lentamente que o dimenidrinato, levando cerca de 3 dias em comparação com 1 dia do dimenidrinato (Rodrigues, et al., 2019).

Em outra pesquisa estudada, o uso da aromaterapia com óleo essencial do gengibre, que feito em uma compressa de gaze resulta em aproximadamente $67 \%$ no alivio de náuseas, em comparação com o grupo placebo que foi de $40 \%$, entretanto uma mistura feita de óleo essenciais, feito com gengibre, hortelã, pimenta e cardomomo, apresentou em uma melhora de $82 \%$ dos pacientes (Rodrigues, et al., 2019).

Já o estudo de Heitmann, et al., (2013) foi possível observar que mulheres que fizeram o uso do gengibre durante a gestação não apresentaram risco de má formação congênita ou baixo peso ao bebê nascer ou parto pré-maturo.

Alguns estudos mostram que o uso de gengibre, administrado por via oral, foram mais eficazes que o grupo placebo na redução da frequência de vômitos e nas náuseas, como também não há risco de qualquer anomalia congênitas. Entretanto, ainda existe algumas incertezas quanto a dosagem segura do gengibre, assim como pode haver consequências se houve super dosagens. Algumas instituições, considera um tratamento alternativo o uso do gengibre, pois ainda é preciso mais estudos para ter certeza da sua segurança (Holand, 2017).

A seguir na Tabela 2 estão listados alguns estudos realizados a fim de avaliar o potencial terapêutico do gengibre na gestação.

Tabela 2. Estudos utilizando gengibre a fim de avaliar seu uso na gestação.

\begin{tabular}{|l|l|}
\hline \multicolumn{1}{|c|}{ Referência } & \multicolumn{1}{|c|}{ Resultado e Conclusão } \\
\hline $\begin{array}{l}\text { SHARIFZADEH, F.; } \\
\text { KASHANIAN, M.; } \\
\text { KOOHPAYEHZADEH, J.; } \\
\text { REZAIAN, F.; } \\
\text { SHEIKHANSARI, N.; } \\
\text { ESHRAGHI, N. (2018). }\end{array}$ & $\begin{array}{l}\text { Apresentou forte ação do uso do gengibre para a diminuição de náuseas e } \\
\text { vômitos. }\end{array}$ \\
\hline $\begin{array}{l}\text { BOLTMAN-BINKOWSKI, H. } \\
\text { (2016). }\end{array}$ & $\begin{array}{l}\text { O estudo apresentou que o uso do gengibre não apresentou nenhum efeito } \\
\text { teratogênico. }\end{array}$ \\
\hline $\begin{array}{l}\text { BALBONTÍN, Y. M.; } \\
\text { STEWART, D.; SHETTY, A.; } \\
\text { FITTON, C. A.; MCLAY, J. S. } \\
\text { (2019). }\end{array}$ & \begin{tabular}{l} 
Não apresentou efeitos adversos decorrente do uso do gengibre. \\
\hline SILVA, M. G. (2015).
\end{tabular} \\
& $\begin{array}{l}\text { Esse estudo mostrou que o gengibre tem uma potente ação antiemética, mas ele } \\
\text { também apresenta efeito anticoagulante, com isso não deve ser usado no final da } \\
\text { gravidez para evitar hemorragias. }\end{array}$ \\
\hline
\end{tabular}

Fonte: Adaptado (Rocha, et al., 2021). 
Outro estudo observado, foi o estudo da DEHON- farmácia de manipulação, onde estudaram o uso do gengibre e sua ação anti-emética eficaz na gravidez e pós-cirúrgico no qual, o objetivo da farmácia neste estudo foi observar se o xarope com gengibre é eficaz no alivio de sintomas no primeiro trimestre da gravidez. Os pesquisadores realizaram o estudo com 26 mulheres e as dividiram em dois grupos: Grupo 1: Gengibre + água; Grupo 2: placebo. O resultado após 9 dias, foi que $77 \%$ das mulheres que recebem o gengibre apresentaram uma melhora significativa no quadro de náuseas e $70 \%$ das mulheres do grupo placebo apresentam pouca ou nenhuma melhora.

Algumas das contra-indicações ao uso do gengibre, está associada a um aumento do sangramento vaginal, incluindo, após a $17^{\mathrm{a}}$ semana de gestação o aparecimento de manchas, entretanto, a associação não foi significativa com o sangramento grave. Como o gengibre pode inibir a síntese de tromboxano e a agregação plaquetária in vitro, existe a preocupação de que o uso muito próximo ao trabalho de parto, possa aumentar o risco de hemorragia pós-parto. Por isso, precisa-se ter alguns cuidados especiais ao fazer o uso do gengibre pois, em doses maiores que $5 \mathrm{~g}$ por dia podem aumentar o risco de efeitos colaterais. Alguns desses efeitos colaterais do gengibre são apresentar desconforto abdominal, azia e diarréia. Alguns dos sintomas gastrointestinais leves, pode ser reduzido pela ingestão do gengibre encapsulado (Rodrigues, et al., 2019).

O gengibre também pode apresentar interações medicamentosas e toxicidade. Em quantidades excessivas, o gengibre pode aumentar o risco de sangramento quando usadas com drogas anticoagulantes ou antiplaquetárias, alguns anticoagulantes ou antiagregantesplaquetários incluem aspirina, clopidogrel (Plavix), dalteparina (Fragmin), enoxaparina (Lovenox), heparina, ticlopidina (Ticlid), varfarina (Coumadin) entre outros, e com isso aumentar a interação medicamentosa (Rodrigues, et al., 2019).

Já a toxicidade do gengibre foi examinada em pesquisas com animais e na pesquisa disponível, não foi possível identificar efeitos tóxicos quando gengibre é administrado por 3 meses. Nos animais que foi administrado pó de gengibre 500, 100 ou $2000 \mathrm{mg} / \mathrm{kg}$ do peso corporal, diariamente durante 35 dias, não foi possível observar associação com a mortalidade ou anomalias desses animais. As gestantes não devem consumir mais que $1000 \mathrm{mg}$ de gengibre por dia durante a gestação, devido ao seu efeito mutagênicos e antiplaquetários, que está associado a doses maiores (Rodrigues, et al., 2019).

Em todas as pesquisas estudadas, o Zingiberofficinale (gengibre) foi o mais citado, se destacando com ótimos resultados bem como, se mostrou altamente eficaz conta os problemas gastrointestinais. O gengibre, apresentou o único recurso fitoterapêutico cuja sua eficiência e segurança contra náuseas e vômitos foram comprovados por diversos estudos clínicos como também, não foram capazes de identificar efeitos teratogênicos em gestantes durante o seu uso, desde que usados corretamente (Rocha, et al., 2021; Cardoso, et al., 2019).

\section{Conclusão}

Há uma falsa idéia que a utilização de plantas medicinais não provoca danos a saúde, hoje sabemos que isto não é uma realidade. O uso inadequado de fitoterápicos requer atenção, principalmente durante a gravidez, sendo necessário ressaltar a importância de campanhas informativas de teor científico para levar informação a população como medida de promoção a saúde.

De acordo com os resultados obtidos, a planta mais citada para uso na gestação contra as náuseas e os vômitos foi o Zingiberofficinale (gengibre). Os dados científicos registrados sobre suas atividades terapêuticas se mostraram bastante eficazes, onde foram alcançados resultados satisfatórias na redução das náuseas e dos vômitos durante o primeiro trimestre da gestação, sem causar quaisquer efeitos teratogênicos e com isso sendo cada vez mais recomendada a sua utilização como uma opção terapêutica. Entretanto, ainda são poucas as pesquisas experimentais e laboratoriais para comprovação da eficácia do uso 
do gengibre, o que evidencia a importância de novas pesquisas, para garantir a validação segura em órgãos reguladores, garantindo por vez sua confiabilidade, já que estamos falando da segurança da mãe e do feto.

\section{Referências}

Amorin, A., Ferreira, A. R. R., \& Carrapiço, E. (2013). Gengibre no tratamento da náusea e vômito da gravidez: revisão baseada na Evidência. Acta ObstetGinecolPort, 7(2), 103-108

Balbontín, Y. M., Stewart, D., Shetty, A., Fitton, C. A., \&McLay, J. S. (2019). Herbal Medicinal Product Use DuringPregnancyandthePostnatalPeriod: A SystematicReview. ObstetGynecol, 133(5), 920-932.

Bode, A. M., \&Dong, Z. (2011). O incrível e poderoso gengibre. 2. ed.CRC Press / Taylor \& Francis. Boca Raton.

Boltman-Binkowski, H. (2016). A systematicreview: Are herbalandhomeopathicremediesusedduringpregnancy safe? Curationis, 39(1).

Cardoso, B. S., \& Amaral, V. C. S. (2017). O uso da fitoterapia durante a gestação: um panorama global. Curso de ciências aplicadas a produtos para a saúde, Campus Anápolis de Ciências Exatas e Tecnológicas, Universidade Estadual de Goiás, Anápolis- Go.

Carvalho, N. S., Bezerra, A. N., Viana, A. C. C., Morais, S. R., \& Azevedo, D. V. (2020). Percepção de gestantes quanto ao uso de plantas medicinais e fitoterápicos: Uma revisão integrativa da literatura. Braz. J. Hea. Rev, 3(4), 9282-9298.

Conceição, K. P. P. (2019). Efeito anti-inflamatório do gengibre. Dissertação (Mestrado) - Ciências farmacêuticas, Instituto universitário Egas Moniz, Portugal.

Conceição, S. F. S. M. (2013). Efeitos do Gengibre, do Alho e do Funcho na Saúde. Projeto de Pós-graduação, ciências da saúde, centro de Ciências Farmacêuticas, Universidade Fernando Pessoa, Porto.

Cutrim, E. S. M., Teles, A. M., Mouchrek, A. N., Mouchrek Filho, V. E., \& Everton, G. O. (2019). Avaliação da Atividade Antimicrobiana e Antioxidante dos Óleos Essenciais e Extratos Hidroalcoólicos de Zingiberofficinale (Gengibre) e Rosmarinusofficinalis (Alecrim). Rev. Virtual Quim, 11(1), 60-81.

Duarte, A. F. S., Martins, A. L. C., Miguel, M. D., \& Miguel, O. G. (2017). O uso de plantas medicinais durante a gravidez e amamentação. Curso de Farmácia, Departamento de Farmácia, Universidade Federal do Paraná, Curitiba.

Figueredo, C. A., Gurgel, I. G. D., \& Junior, G. D. G. (2014). A política nacional de plantas medicinais e fitoterápicos: construção, perspectivas e desafios. Physis Revista de Saúde Coletiva, 24(2), 381-400.

Gomes, M. B. A., Galindo, E. A., \& Lins, S. R. O. (2018). Uso de plantas medicinais durante o período gestacional: uma breve revisão. Braz. J. Hea. Rev, $1(2), 323-327$.

Gomes, B. S. S. (2018). Relatório de estágio em farmácia comunitária e monografia intitulada "Plantas medicinais na gravidez". Curso de Farmácia, Mestrado Integrado em Ciências Farmacêuticas, Universidade de Coimbra, Coimbra.

Hemkemeier, D., Chamusca, G., Carnevali, L. C., Gonçalves, D. C., \&Hoefel, A. L. (2018). Uso de gengibre pode ser efetivo em diminuir sintomas de náuseas na gestação: uma revisão. Revista Científica Multidisciplinar Núcleo do Conhecimento, 10(8), 101-112.

Holand, B. L. (2017). Efeitos do gengibre sobre náusea e êmese na gestação: uma revisão sistemática. Curso de Nutrição, Departamento de Nutrição, Universidade Federal do Rio Grande do Sul, Porto Alegre.

Malagoli, B. G., Nascimento, M. M. G., Rezende, C. P., \& Silva, D. Á. M. (2019). Uso seguro de medicação na gestação. Boletim, instituto para práticas seguras no uso de medicamentos, 8(10), 1-14.

Neto, G. A. (2018). GENGIBRE (Zingiberofficinale) ação anti-emética eficaz na gravidez e pós-cirúrgico. DEHON- Farmácia de Manipulação. https://docplayer.com.br/26246166-Gengibre-zingiber-officinale-acao-anti-emetica-eficaz-na-gravidez-e-pos-cirurgico.html.

Pereira, A. S., Shitsuka, D. M., Parreira, F. J., \& Shitsuka, R. (2018). Metodologia da pesquisa científica. [free e-book]. Santa Maria/RS. Ed.UAB/NTE/UFSM. https://www.ufsm.br/app/uploads/sites/358/2019/02/Metodologia-da-Pesquisa-Cientifica_final.pdf.

Pires, A. M., \& Araújo, P. S. (2011). Percepção de risco e conceitos sobre plantas medicinais, fitoterápicos e medicamentos alopáticos entre gestantes. Revista Baiana de Saúde Pública, 35(2), 320-333.

Rocha, F. S., Santos, J. V. C., Resende, R. O., Santos, T. B., Junior, E. C. A., Almeida, D. D., Santos, D. M. S., \&Gallotti, F. C. M. (2021). Uso de fitoterápicos como alternativa para a diminuição da sintomatologia recorrente na gravidez.Res., Soc. Dev, 10(3), 1-12.

Rodrigues, E. S., Ferreira, I. P., \& Andrade, M. A. (2019). Protocolo de plantas medicinais e fitoterápicos na assistência obstétrica. Dissertação (Mestrado) Curso de Gestão e Saúde, Fundação Santa Casa de Misericórdia do Pará, Belém.

Sharifzadeh, F., Kashanian, M., Koohpayehzadeh, J., Rezaian, F., Hheikhansari, N., \&Eshraghi, N. (2018). A comparisonbetweentheeffectsof ginger, pyridoxine (vitamin B6) and placebo for thetreatmentofthefirsttrimesternauseaandvomitingofpregnancy (NVP). J Matern Fetal Neonatal Med, 31(19), 25092514.

Silva, M. G. (2015). Plantas Medicinais na Gravidez e Aleitamento. Monografia (Especialização) - Curso de Farmácia, Estágio Curricular do Mestrado Integrado em Ciências Farmacêuticas, Universidade de Coimbra, Coimbra.

Vieira, N. A., Tomiotto, F. N., Melo, G. P., Manchope, M. F., Lima, N. R., Oliveira, G. G., \& Watanabe, M. A. E. (2014). Efeito anti-inflamatório do gengibre e possível via de sinalização. Semina: Ciências Biológicas e da Saúde, 35(1), 149-162. 